\title{
MODIFIED LIGNIN AS AN ENVIRONMENTALLY FRIENDLY SURFACTANT
}

\author{
G. Shulga ${ }^{1}$, V. Shakels ${ }^{1}$, S. Skudra ${ }^{1}$, V. Bogdanovs ${ }^{2}$ \\ 1- Latvian State Institute of Wood Chemistry, 27 Dzerbenes Str., LV 1006, Riga, Latvia \\ Ph.: (+371) 67545139, e-mail: shulga@junik.lv; vadim@ saint-tech.lv; \\ sanita.skudra@gmail.com \\ 2- SIA “JUSMA”, Citadeles 2-623, LV 1010, Riga, Latvia
}

\begin{abstract}
The effect of softwood sulphate lignin modified by its oxidation in alkaline medium on its behaviour at the air-water and oil-water interface, where rapeseed oil and technical fish fat oil served as the oil phase, was investigated. It has been found that, irrespective of the $\mathrm{pH}$ values of the water solution, the ability of the modified lignin to be adsorbed at the interfaces exceeds that of unmodified lignin, which is obviously caused by the pronounced differentiation of the hydrophilic and hydrophobic parts of the molecular structure in the oxidised lignin and the decrease in the content of the high-molecular fraction $\left(M_{w}>1000\right)$ in it. At low concentrations $(\leq 0.01 \mathrm{~g} / \mathrm{dl})$, the stabilising action of the modified lignin in the rapeseed oil-water emulsion is the same as that of sodium dodecyl sulphate. The enhanced surface activity of the oxidised sulphate lignin enables it not only to substitute the $30 \%$ of the mass of the commercial surfactant in the detergent composition for washing stainless steel plates, used during fish smoking, but also to upgrade its properties.
\end{abstract}

Keywords: detergent composition, fish smoking, modified lignin, oil-in-water emulsion, stabiliser, surface tension.

\section{Introduction}

Due to the increasing requirements to environmental protection, obtaining of "green" surfactants from natural feedstock represents both scientific and practical interest. With this target, it is necessary to use renewable low-cost materials that are available in large quantities and to modify their molecular structures that show improved performance, favourable ecotoxicological properties and reduced environmental impact [1]. The surfactants from byproducts of the sugar and oleochemical industries or derived from sea sources are well known [2-3]. Technical lignins, by-products of the industrial wood processing, have found widespread application as an available renewable bioresource for producing different commercially attractive end-products, including dispersants, detergents and surfactants [4]. Lignins, due to the peculiarities of their aromatic structure, can easily adsorb at various interfaces, creating mono- or multi-interface layers, which provide a decrease of interfacial tension. For obtaining "green" surfactants, lignins are chemically modified, using various methods of alkylation, sulphonation, destruction, etc. [5, 6].

In the present study, the effect of the oxidation of technical lignin, a commercial by-product, on its surface active properties at the gas-liquid and liquid-liquid interfaces, as well as its possibility to replace the surface active agent in the commercial detergent composition, which is used for cleaning stainless steel plates in fish smoking, has been investigated. It is known that Latvian fish processing is an export-oriented sector, and one of its export productions is smoked fish.

\section{Materials and methods}

Commercial softwood sulphate lignin (SL) in the form of a high-dispersed brown powder was decontaminated from a strong alkaline solution $(\mathrm{pH} 13.3)$ by precipitation with sulphuric acid to $\mathrm{pH} 2$ in accordance with the method [7]. The modification of SL was carried out in alkaline medium at $60^{\circ} \mathrm{C}$ in the presence of air oxygen under continuous stirring for $1 \mathrm{~h}$. The detailed procedure of its modification with the following drying is described in our previous work [8]. 
The initial sample and that modified by oxidation, called as SL and SL-1, were subjected to chemical analyses. The elemental and functional analysis of the lignin samples was performed, applying the traditional analytical methods [9], including potentiometric and conductometric titration (InoLab level 3, Germany). The chemical composition of the initial and modified lignin is given in Table 1.

Table 1.

Effect of modification on the elemental and functional composition of SL

\begin{tabular}{|c|c|c|c|c|c|c|c|c|c|}
\hline \multirow[t]{2}{*}{$\begin{array}{l}\text { Ligni } \\
\text { n }\end{array}$} & \multirow[t]{2}{*}{$\begin{array}{l}\mathrm{C}, \\
\%\end{array}$} & \multirow[t]{2}{*}{$\begin{array}{l}\mathrm{H}, \\
\%\end{array}$} & \multirow[t]{2}{*}{$\begin{array}{l}\text { O, } \\
\%\end{array}$} & \multirow[t]{2}{*}{$\begin{array}{l}\text { S, } \\
\%\end{array}$} & \multirow[t]{2}{*}{$\underset{\%}{\mathrm{OCH}_{3},}$} & \multirow{2}{*}{$\underset{\%}{\mathrm{CO}}$} & \multirow[t]{2}{*}{$\begin{array}{c}\mathbf{O H}_{\text {total }}, \\
\%\end{array}$} & \multicolumn{2}{|c|}{$\begin{array}{c}\text { Content of } \\
\text { functional } \\
\text { groups, meqv/g }\end{array}$} \\
\hline & & & & & & & & $\mathbf{O H}_{\mathrm{ph}}$ & $\mathrm{COOH}$ \\
\hline SL & 63.9 & 5.5 & 27.9 & 2.7 & 12.2 & 3.5 & 9.7 & 5.1 & 5.4 \\
\hline SL-1 & 49.7 & 4.7 & 43.2 & 2.4 & 7.4 & 3.2 & 7.3 & 3.5 & 6.7 \\
\hline
\end{tabular}

The molecular mass (M) parameters such $\left(\mathrm{M}_{\mathrm{z}}, \mathrm{M}_{\mathrm{w}}\right.$ and $\left.\mathrm{M}_{\mathrm{n}}\right)$ of the lignin samples, represented in Table 2, were determined by the SEC method, using a liquid chromatograph (Gilson, Middleton, U.S.A.). Based on the elemental and functional composition data, the average weight of the phenyl propane unit (PPU) for each sample was calculated.

The found values of molecular masses for SL and SL-1 are given in Table 2. The surface tension $(\sigma)$ at the air-water interface was measured by the Wilhelmy method at $25.0 \pm 0.1{ }^{\circ} \mathrm{C}$, using a tensiometer K9 (KRUSS, Germany) and a circular thermostat TC-102 (Brookfield, $\mathrm{UK})$. The values of $\sigma$ were determined for each concentration and $\mathrm{pH}$ value of the lignin aqueous solution after $24 \mathrm{~h}$ storing at room temperature. The solutions of the lignin samples were obtained by diluting the more concentrated solutions in order to reduce errors in weighing.

Table 2.

SL and SL-1 molecular mass characteristics

\begin{tabular}{|l|c|c|c|c|c|c|}
\hline Lignin & $\mathbf{M}_{\mathbf{z}}$ & $\mathbf{M}_{\mathbf{w}}$ & $\mathbf{M}_{\mathbf{n}}$ & $\mathbf{M}_{\mathbf{w}} / \mathbf{M}_{\mathbf{n}}$ & $\begin{array}{c}\text { Content of } \\
\text { high- } \\
\text { molecular } \\
\text { fraction } \\
\left.\mathbf{M}_{\mathbf{w}}>\mathbf{1 0 0 0}\right), \\
\mathbf{\%}\end{array}$ & PPU \\
\hline SL & 6020 & 4160 & 2840 & 1.47 & 70.1 & 178 \\
SL-1 & 8080 & 5550 & 3700 & 1.50 & 62.2 & 225 \\
\hline
\end{tabular}

The $\mathrm{pH}$ values were regulated by adding $1 \mathrm{M} \mathrm{HCl}$ and $1 \mathrm{M} \mathrm{NaOH}$. The stabilising effect of the lignin samples as well as the detergent composition in terms of the volume $(\mathrm{H})$ and time $(\mathrm{t})$ of water separation was studied in the rapeseed oil-in-water emulsion $(40 / 60, \mathrm{v} / \mathrm{v})$ and the fish fat oil-in-water one $(20 / 80, v / v)$, prepared with a Disperser T10 (IKA, Germany) $(\gamma=9500$ $\mathrm{rpm})$ for $1 \mathrm{~min}$. The fish fat oil represented a by-product of the smoking process at fish working enterprises.

For the study of the ability of the modified lignin to replace the surfactant in the detergent composition, which is commonly used for cleaning stainless steel plates in fish processing, the following commercial detergent composition, containing the surfactant 5\%, sodium gluconate $2 \%$, phosphate $1 \%, \mathrm{NaOH} 40 \%$ and a distilled water $52 \%$, was used. The detergent composition, in which $30 \%$ of the surfactant was replaced by the modified lignin, was prepared for comparing the stabilising action of both surfactants to the oil-in-water emulsions. The time of observation was $2 \mathrm{~h}$. Each experiment was performed at least 3 times, and the results were represented as an arithmetic average. 


\section{Results and discussion}

Table 1 shows that, after $1 \mathrm{~h}$ of the alkaline modification, the degree of oxidation $(\mathrm{O} / \mathrm{C})$ of SL-1 increases twice in comparison with that of the initial lignin. Simultaneously, the content of the methoxyl and phenolic hydroxyl groups decreases by $64 \%$ and $33 \%$, respectively, but the content of the carboxylic groups increases by $25 \%$. In spite of the negligible growth of the molecular masses of SL-1 relative to SL (Table 2), the content of the high molecular fraction in the modified lignin decreases. The relatively minor alteration in the average molecular mass parameters against the background of the considerable change in the chemical composition, taking into account the "core-shell" structure of lignin [10], may indicate that, during the oxidation process, the main chemical transformations in the SL macromolecules occur at the "shell" chains level.

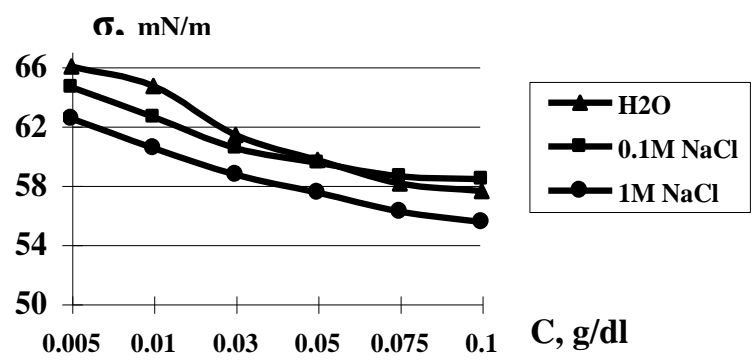

a

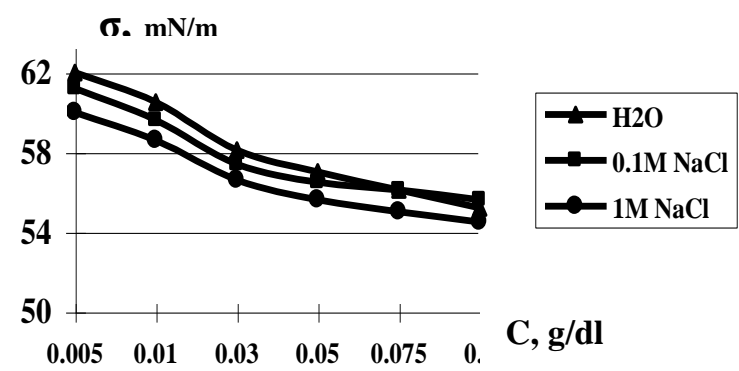

b

\section{Fig. 1. Surface tension at the air-water interface of SL (a) and SL-1 (b) water solutions with different ionic strength at $\mathrm{pH} 12.0$ vs. concentration}

According to Figure 1, the surface tension at the air-water interface of both the initial and modified lignin aqueous solutions decreases with the growth in the concentration of the lowmolecular electrolyte in alkaline medium. This indicates the increase in the hydrophobicity of the lignin molecules as a result of the decrease in the Stern potential and the compression in the thickness of the double electric layer around the lignin macromolecules. It can be seen (Fig. 1) that SL-1 is characterised by the lowest values of $\sigma$, while the initial lignin has the largest values of the surface tension both in a salt-free medium and in the presence of salts.

The simultaneous presence of the pronounced aromatic hydrophobic fragments and hydrophilic ones, containing the enhanced amount of carboxylic group, in SL-1 at its relatively higher content of the low molecular fractions $\left(M_{w}<1000\right)$ governs the high tendency of the oxidised lignin to self-aggregation and self-organisation at the interface layers, resulting in more dense packing of the lignin macromolecules in them, leading to lowering the surface tension. According to Table 2, the values of the molecular mass parameters for the initial and modified lignin differ negligibly. Hence, the main reason, influencing the favourable joint orientation and re-assembling, as well as the quality of packing at the interface may be the pronounced differentiation of the hydrophilic and hydrophobic fragments in the structure of SL-1. 


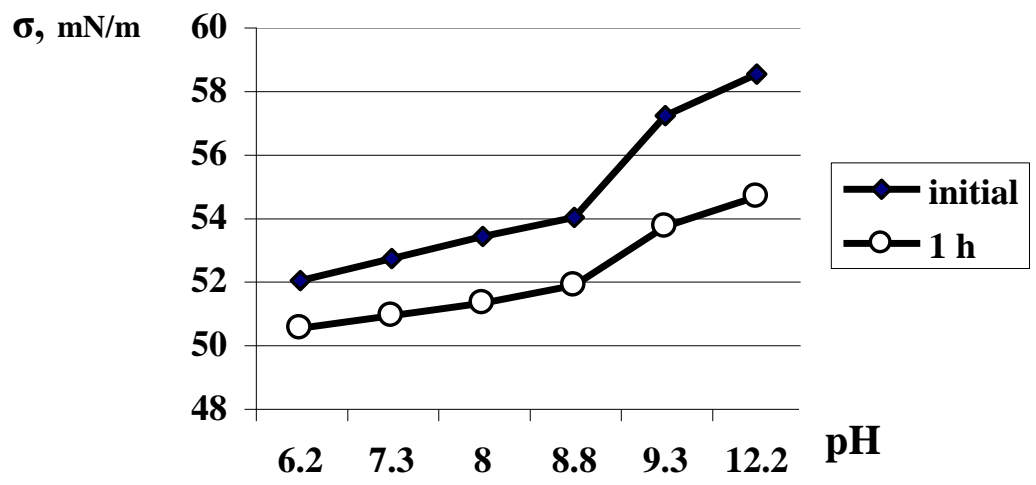

Fig. 2. Surface tension at the air-water interface of SL (a) and SL-1 (b) water solutions vs. $\mathrm{pH}$ values; concentration $-0.1 \mathrm{~g} / \mathrm{dl}$

The surface tension of both lignin samples decreases with decreasing pH (Fig. 2), which is caused by the hydrophobisation of their structure as a result of the protonisation of the phenolic hydroxyl and carboxyl groups, promoting the association of the lignin macromolecules both in solution and at the interface. At the same time, independently on $\mathrm{pH}$, the oxidised lignin is characterised by lower values of the surface tension at the air-water interface in comparison with the unmodified lignin.
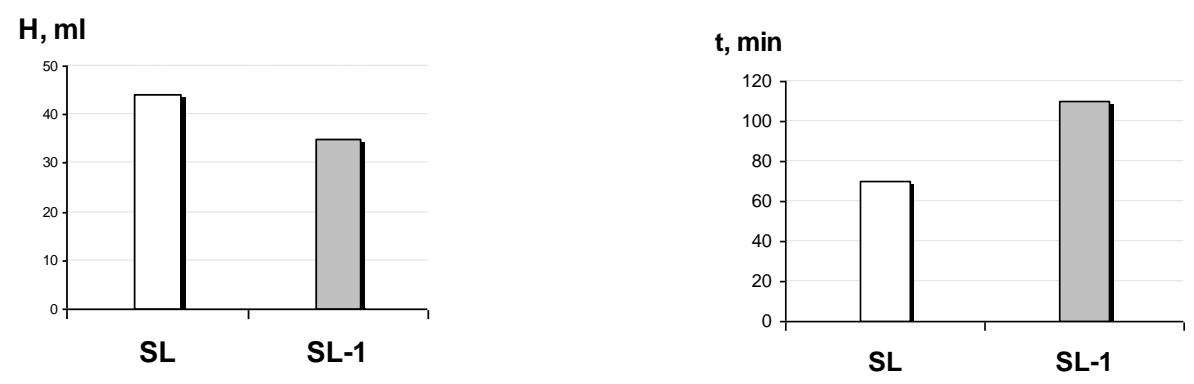

Fig. 3. Final volume of the water and time separation of the rapeseed oil-in-water emulsion, stabilising with initial and modified lignin; $\mathrm{pH}$ of water phase 12.0; lignin concentration - $0.1 \mathrm{~g} / \mathrm{dl}$

The comparative stabilising effect of SL and SL-1 in terms of the volume and time of water separation was studied in the rapeseed oil-in-water emulsion. It has been found that, with increasing the $\mathrm{pH}$ values of the water phase and growing the concentration of the lignin samples in it, the ability of the lignin samples to be adsorbed at the water-oil interface grows. At the same time, the ability of the SL-1 molecules to stabilise the water-oil emulsion is higher than that of the initial lignin. According to Fig. 3, the time of separation of the emulsion, stabilised by the modified lignin, is higher by 55\%, but the volume of separation is less by $20 \%$ than those in the case of the emulsion, containing the initial lignin. It is known that the formation of interface layers in such a disperse system obviously occurs as a result of the hydrophobic interaction between the surface groups of the oil drops and the stabiliser molecule. 


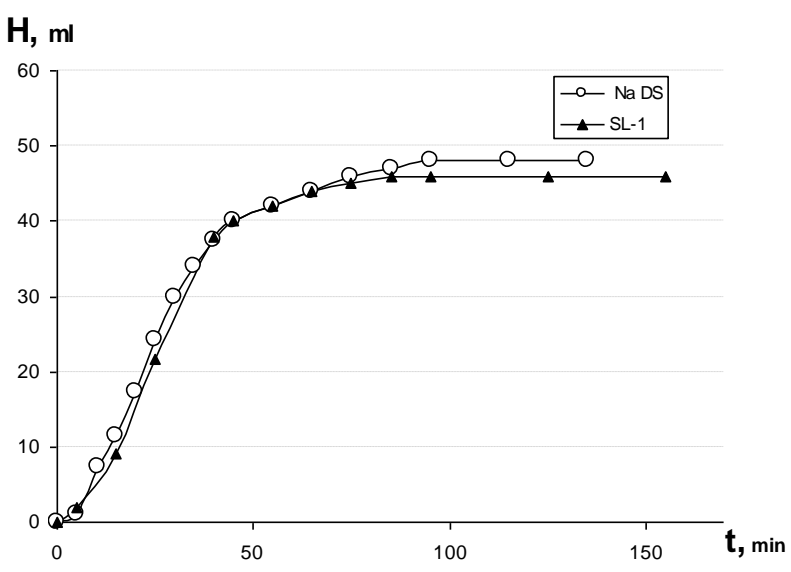

Fig.4. Dependence of the volume of water separation on separation time for the rapeseed oil-in-water emulsion, stabilising with SL-1 and Na-DS sodium dodecyl sulphate; $\mathrm{pH}$ 12.0; concentration $-7.5 \cdot 10^{-3} \mathrm{~g} / \mathrm{dl}$

The pronounced differentiation of the hydrophobic and hydrophilic parts in the SL-1 macromolecule, along with its lower content of high molecular fraction, may promote its favourable transfer and fixation at the oil-water interface, similarly to the case of its adsorption at the water-air interface.

Table 3.

Comparison of the stability of the fish fat oil-in-water emulsion, containing the initial detergent composition and the composition with the modified lignin

\begin{tabular}{|l|c|c|}
\hline Composition & $\begin{array}{c}\text { Final volume of water } \\
\text { separation, ml }\end{array}$ & $\begin{array}{c}\text { Final time of water } \\
\text { separation, min }\end{array}$ \\
\hline Initial detergent composition & 70 & 60 \\
\hline $\begin{array}{l}\text { Composition, in which the } \\
\text { surfactant is partially } \\
\text { replaced by SL-1 (30\% of } \\
\text { its content) }\end{array}$ & 64 & 120 \\
\hline
\end{tabular}

It is known that sodium dodecyl sulphate (Na-DS) is a highly effective surfactant, which is used for the removal of oily stains and residues [11]. Fig. 4 demonstrates separation curves of the rapeseed oil-in-water emulsion for Na-DS and ML. The content of the lignin samples in the water phase is less than $0.01 \mathrm{~g} / \mathrm{dl}$, which is typical for the used working concentrations of the synthetic surfactants. It can be seen that, at the given content in the water phase, the stabilising action of Na-DS and SL-1 is practically the same, which is confirmed by the equal values of the time and volume of separation in the water-in-oil emulsion. It is known that fish smoking releases a fish fat oil, which contaminates the equipment, including the used stainless steel plates. For fish fat oil removal, synthetic surfactants are commonly used, but their impact on the environment does not always comply with the required demands. In addition, with each year, the synthetic surfactant prices increase due to the growth of the oil price in the world.

Taking this into account, an attempt was made to replace a part of the commercial surfactant by the modified lignin in the detergent composition. According to the data listed in Table 3, the partial substitution of the used surfactant with the modified lignin enables not only the equivalent substitution, but also the considerable upgrading of the properties of the commercial detergent composition, which is testified by the two-fold increase in the value of the separation time of the fish fat oil-in-water emulsion, containing the modified lignin. 


\section{Summary}

In the present study, the influence of the softwood sulphate lignin, modified by oxidation in alkaline medium, on its surface active properties at the gas-liquid and liquid-liquid interfaces was investigated. Irrespective of the $\mathrm{pH}$ values of the water solution, the ability of the modified lignin to be adsorbed at the interfaces exceeds that of unmodified lignin both in a salt-free water solution and in the presence of sodium chloride. The enhanced surface activity of the modified lignin enables it not only to substitute the $30 \%$ of the synthetic surfactant in the detergent composition for washing stainless steel plates, used during fish smoking, but also to upgrade its stabilizing ability.

\section{Acknowledgement}

The researches leading to these results have received funding from the Latvian Council of Science for a grant $\mathrm{n}^{\circ} 09-1610 \mathrm{c}$, as well as from the funding of the "WOOD-NET" project of FP7/2007-2013 (agreement n²03459).

\section{References}

1. Benvegnu, T., Plusquellec, D., Lemiègre, L. Monomers, polymers and composites from renewable resources. Elsevier, Amsterdam, 2008.

2. Roussel, M., Benvegnu, T., Lognoné, V., Le Deit, H., Soutrel, I., Laurent, I., Plusquellec, D. Synthesis and physico-chemical properties of novel biocompatible alkyl d-mannopyranosiduronate surfactants derived from alginate. Eur. J. Org. Chem., 14, 2005, p. 3085-3094.

3. Goursaud, F., Berchel, M., Guilbot, J., Legros, N., Lemiègre, L., Marcilloux, J., Plusquellec, D., Benvegnu, T. Glycine betaine as a renewable raw material to „greener” new cationic surfactants. Green Chem., 10 (3), 2008, p. 310-320.

4. Sarkanen, K.V., Ludwig, C.H. (Eds.). Lignins: occurrence, formation, structure and reactions. John Wiley \& Sons, New York, 1971.

5. Moeller, T., Soldanski, H-D., Kuech, S., Noglich, J. Multiphase cleaning composition containing lignin sulfonate. U.S. Patent 6,288,015, 2001.

6. Yang, D., Qiu, X., Zhou, M., Lou, H. Properties of sodium lignosulfonate as dispersant of coal water slurry. Energy Conversion and Management, 48(9), 2007, p. 2433-2438.

7. Lin, S.Y., Dence, C.W. (Eds.). Methods in lignin chemistry. Springer-Verlag, Berlin-Heidelberg, 1992, p. 568.

8. Shulga, G., Bikova, T., Priede, V., Treimanis, A. Chemical transformations in kraft-lignin matrix during long-term alkali oxidation. Chemical Technology, 1(35), 2005, p. 80-84.

9. Zakis, G.F. Functional analysis of lignins and their derivatives. Tappi Press, Atlanta, 1994.

10. Shulga, G., Priede, V., Shakels, V., Bikova, T., Treimanis, A. Behavior of oxidized kraft lignin as a "coreshell" polymer in aqueous solutions. Abstracts of the $39^{\text {th }}$ IUPAC Congress "Chemistry at the Interfaces" and the $86^{\text {th }}$ Conference of the Canadian Society for Chemistry. Ottawa, Canada, 10-15 August, 2003, p. 271.

11. Sodium lauryl sulfate [retrieved on 14.02.2011], website: http://en.wikipedia.org/wiki/Sodium-laurylsulfate. 